\title{
LOCAL ISOMETRIES OF COMPACT METRIC SPACES
}

\author{
ALEKSANDER CALKA
}

\begin{abstract}
By local isometries we mean mappings which locally preserve distances. A few of the main results are:

1. For each local isometry $f$ of a compact metric space $(M, \rho)$ into itself there exists a unique decomposition of $M$ into disjoint open sets, $M=M_{0}^{f} U$ $\cdots \cup M_{n}^{f},(0 \leq n<\infty)$ such that (i) $f\left(M_{0}^{f}\right)=M_{0}^{f}$, and (ii) $f\left(M_{i}^{f}\right) \subset M_{i-1}^{f}$ and $M_{i}^{f} \neq 0$ for each $i, 1 \leq i \leq n$.

2. Each local isometry of a metric continuum into itself is a homeomorphism onto itself.

3. Each nonexpansive local isometry of a metric continuum into itself is an isometry onto itself.

4. Each local isometry of a convex metric continuum into itself is an isometry onto itself.
\end{abstract}

1. Introduction. A mapping $f$ of a metric space $(M, \rho)$ into a metric space $(N, \delta)$ is said to be a local isometry if for each $z \in M$ there exists a neighborhood $U$ of $z$ such that

$$
\delta(f(x), f(y))=\rho(x, y) \text { for all } x, y \in U
$$

Mappings, as above, satisfying (1) with $U=M$ are called isometries.

The present paper concerns local isometries of compact metric spaces into itself. In $\S 3$ we collect the necessary information concerning locally nonexpansive mappings. In $\S 4$ we prove the main result: For each local isometry $f$ of a compact metric space $(M, \rho)$ into itself, there exists a unique decomposition of $M$ into disjoint open sets, $M=M_{0}^{f} \cup \cdots \cup M_{n}^{f}$, such that (i) $f\left(M_{0}^{f}\right)=M_{0}^{f}$, (ii) $f\left(M_{i}^{f}\right) \subset$ $M_{i-1}^{f}$ and $M_{i}^{f} \neq \emptyset$ for each $i, 1 \leq i \leq n$. Moreover, there is a metric on $M$, topologically equivalent to $\rho$, with respect to which $f$ is a nonexpansive local isometry and maps $M_{0}^{f}$ isometrically onto itself. $\$ 5$ contains some consequences of the main result. Namely, we prove: Each local isometry of a metric continuum into itself is a homeomorphism onto itself. Each nonexpansive local isometry of a metric continuum into itself is an isometry onto itself. Each local isometry of a convex metric continuum into itself is an isometry onto itself.

It should be mentioned that open surjective local isometries were studied by Busemann [2] (cf. also [3]), Kirk [6] and Szenthe [10], in the special case where $(M, \rho)$ is a $G$-space (Busemann [2] called them "locally isometric mappings"). Busemann [2, (27.14)] proved that every open local isometry of a compact $G$-space onto itself is an isometry. Thus (5.5) of the present paper generalizes this result to the case of general local isometries of convex metric continua.

Received by the editors January 5, 1981.

1980 Mathematics Subject Classification. Primary 54H20, 54E40.

Key words and phrases. Locally nonexpansive mapping, nonexpansive mapping, local isometry, isometry, decomposition of the space, convex space. 
2. Some preliminaries. The following definition is introduced:

(2.1) DEFINITION. Let $\rho_{i}, i=0,1$, be metrics on a set $M$. We shall say that $\rho_{1}$ is locally identical with $\rho_{0}$ if the identity mapping, $\mathrm{id}_{M}$, of $M$ is a local isometry of the metric space $\left(M, \rho_{0}\right)$ into the metric space $\left(M, \rho_{1}\right)$. We shall say that $\rho_{1}$ and $\rho_{0}$ are locally identical if $\rho_{i}$ is locally identical with $\rho_{j}$, for all $i, j=0,1$.

(2.2) REMARKS. Let $\rho_{i}, i=0,1$, be metrics on a set $M$. If $\rho_{1}$ and $\rho_{0}$ are locally identical then they are topologically equivalent. If the metric space $\left(M, \rho_{0}\right)$ is compact and $\rho_{1}$ is locally identical with $\rho_{0}$ then $\rho_{1}$ and $\rho_{0}$ are locally identical (and topologically equivalent). If $f$ is a local isometry of $\left(M, \rho_{0}\right)$ into itself and $\rho_{1}$ and $\rho_{0}$ are locally identical then $f$ is a local isometry of $\left(M, \rho_{1}\right)$ into itself.

(2.3) Definition. A mapping $f$ of a metric space $(M, \rho)$ into a metric space $(N, \delta)$ is said to be locally nonexpansive if for each $z \in M$ there exists a neighborhood $U$ of $z$ such that

$$
\delta(f(x), f(y)) \leq \rho(x, y) \text { for all } x, y \in U .
$$

Mappings, as above, satisfying (2) with $U=M$ are called nonexpansive.

(2.4) REMARKS. (a) Local isometries (isometries) are locally nonexpansive (nonexpansive) mappings.

(b) Let $f$ be a local isometry (a locally nonexpansive mapping) of a compact metric space $(M, \rho)$ into itself. Then there exists a number $\epsilon>0$ such that for all $x, y \in M$,

$$
\rho(x, y)<\epsilon \text { implies } \rho(f(x), f(y))=\rho(x, y)
$$

(respectively,

$$
\rho(x, y)<\epsilon \text { implies } \rho(f(x), f(y)) \leq \rho(x, y)) .
$$

In the sequel we need the following well-known facts which may be found in [5 and 7] (or in [9]).

(2.5) LEMMA. Each nonexpansive mapping of a compact metric space onto itself is an isometry.

(2.6) LEMMA. Each isometry of a compact metric space into itself is a surjection.

\section{Locally nonexpansive mappings and induced metrics.}

(3.1) DEFINITION. Let $f$ be a mapping of a metric space $(M, \rho)$ into itself. Then the function $\rho_{f}$ defined by $\left(f^{0}=\operatorname{id}_{M}, f^{n+1}=f \circ f^{n}\right)$,

$$
\rho_{f}(x, y)=\sup _{n \geq 0} \rho\left(f^{n}(x), f^{n}(y)\right) \text { for all } x, y \in M,
$$

will be called the induced metric on $M$.

Note. If $f$ is a mapping of a bounded metric space $(M, \rho)$ into itself then the induced metric $\rho_{f}$ is a metric on the set $M$ such that

$$
\rho_{f} \geq \rho
$$

(6) $f$ is a nonexpansive mapping of the metric space $\left(M, \rho_{f}\right)$ into itself,

(7) $\rho_{f}=\rho$ if and only if $f$ is a nonexpansive mapping of $(M, \rho)$ into itself. 
We have

(3.2) LEMMA. Let $f$ be a locally nonexpansive mapping of a compact metric space $(M, \rho)$ into itself. Then the induced metric, $\rho_{f}$, is a metric on $M$ such that $\rho_{f}$ and $\rho$ are locally identical. In particular, $\rho_{f}$ and $\rho$ are topologically equivalent.

Proof. Since $(M, \rho)$ is compact, it is bounded and thus $\rho_{f}$ is a metric on $M$.

Let $\epsilon>0$ be a number satisfying (4). Thus, by induction, $\rho\left(f^{n}(x), f^{n}(y)\right) \leq$ $\rho(x, y)$ for each $n \geq 0$ and all $x, y \in M$ with $\rho(x, y)<\epsilon$. Hence, $\rho_{f}(x, y)=\rho(x, y)$ for all $x, y \in M$ with $\rho(x, y)<\epsilon$, which shows that $\rho_{f}$ is locally identical with $\rho$ and therefore by (5) (or by (2.2)), $\rho_{f}$ and $\rho$ are locally identical.

REMARK. It should be noted that, conversely, if $f$ is a mapping of a bounded metric space $(M, \rho)$ into itself such that the induced metric $\rho_{f}$ is locally identical with $\rho$ then $f$ is locally nonexpansive.

(3.3) PROPOSITION. Let $f$ be a locally nonexpansive mapping of a compact metric space $(M, \rho)$ into itself and let $\rho_{f}$ be the induced metric on $M$. Then $\rho_{f}$ is a metric on $M$ such that $\rho_{f}$ and $\rho$ are locally identical and $f$ is a nonexpansive mapping of $\left(M, \rho_{f}\right)$ into itself, which maps the set $\bigcap_{n \geq 0} f^{n}(M)$ isometrically onto itself.

PrOOF. By (3.2) and (6) we need only show that $f$ maps the set $\bigcap_{n \geq 0} f^{n}(M)$ isometrically onto itself in the metric $\rho_{f}$. However, since the sequence of compact sets $f^{n}(M), n=0,1, \ldots$, is decreasing, hence $f$ maps the compact set $\bigcap_{n \geq 0} f^{n}(M)$ onto itself and our assertion follows from (2.5).

We have the following immediate corollaries of (3.3) (cf. also (2.2)):

(3.4) COROLLARY. Let $f$ be a locally nonexpansive mapping of a compact metric space $(M, \rho)$ onto itself and let $\rho_{f}$ be the induced metric on $M$. Then $\rho_{f}$ is a metric on $M$ such that $\rho_{f}$ and $\rho$ are locally identical and $f$ is an isometry of $\left(M, \rho_{f}\right)$ onto itself.

(3.5) COROLLARY. Each locally nonexpansive mapping of a compact metric space onto itself is a homeomorphism and a local isometry.

4. Decomposition theorem. To show the main result we need two lemmas.

(4.1) LEMMA. If $f$ is an injective local isometry of a compact metric space $(M, \rho)$ into itself, then $f$ is also surjective.

Proof. Let $\epsilon>0$ be a number satisfying (3). Since $M$ is compact, $f$ is a homeomorphism onto a compact subset of $M$, therefore there is a number $\delta>0$ such that for all $x, y \in M$,

$$
\rho(f(x), f(y))<\delta \text { implies } \rho(x, y)<\epsilon .
$$

Let $r=\min \{\delta, \epsilon\}$ and let $\rho^{r}(x, y)=\min \{\rho(x, y), r\}$ for all $x, y \in M$. Thus $\rho^{r}$ is a metric on $M$ topologically equivalent to $\rho$ and it follows from (3) and (8) that $f$ is an isometry of $\left(M, \rho^{r}\right)$ into itself. Our assertion follows now from (2.6).

(4.2) LEMMA. Let $f$ be a local isometry of a compact metric space $(M, \rho)$ into itself. Then the set $B=\bigcap_{n \geq 0} f^{n}(M)$ is an open and closed subset of $M$ and $f(B)=B$. 
PrOOF. By (3.3) (cf. also (2.2)) it is sufficient to prove this in the case where $f$ is a nonexpansive local isometry and maps $B$ isometrically onto itself, which we now assume.

For each $r>0$, let $B(r)=\{x \in M: \rho(x, B)<r\}$. Thus

$$
f(B(r)) \subset B(r) \text { for all } r>0 \text {. }
$$

Let $\epsilon>0$ be a number satisfying (3). We assert that $f$ maps $B(\epsilon / 4)$ injectively into itself.

In fact, let $x, y \in B(\epsilon / 4)$ be such that $f(x)=f(y)$. Then $\rho(x, a)<\epsilon / 4$ and $\rho(y, b)<\epsilon / 4$ for some $a, b \in B$ and by our assumption we have

$$
\begin{aligned}
\rho(a, b) & =\rho(f(a), f(b)) \leq \rho(f(a), f(x))+\rho(f(y), f(b)) \\
& \leq \rho(a, x)+\rho(y, b)<\epsilon / 2 .
\end{aligned}
$$

Thus

$$
\rho(x, y) \leq \rho(x, a)+\rho(a, b)+\rho(b, y)<\epsilon / 4+\epsilon / 2+\epsilon / 4=\epsilon,
$$

and by the definition of $\epsilon, \rho(x, y)=\rho(f(x), f(y))=0$, therefore $x=y$, as desired.

Now, let $0<r<\epsilon / 4$ and let $N$ be the closure of $B(r)$. Thus $N \subset B(\epsilon / 4)$ and by (9), the restriction of $f$ to $N$ is an injective local isometry of $N$ into itself. Since $N$ is compact, it follows from (4.1) that $f(N)=N$. Thus for each $n \geq 0$, $f^{n}(N)=N$, therefore $N \subset \bigcap_{n \geq 0} f^{n}(M)=B$. Hence, by the definition of $N$, $B(r)=N=B$ and therefore $B$ is an open and closed subset of $M$. This completes the proof.

(4.3) THEOREM. Let $f$ be a local isometry of a compact metric space $(M, \rho)$ into itself. Then there exists a unique decomposition of $M$ into disjoint open sets

$$
M=M_{0}^{f} \cup \cdots \cup M_{n}^{f}
$$

such that

$$
\begin{gathered}
f\left(M_{0}^{f}\right)=M_{0}^{f}, \\
f\left(M_{i}^{f}\right) \subset M_{i-1}^{f} \text { and } M_{i}^{f} \neq 0 \text { for each } i, 1 \leq i \leq n .
\end{gathered}
$$

Moreover, the induced metric $\rho_{f}$ is a metric on $M$ such that $\rho_{f}$ and $\rho$ are locally identical and $f$ is a nonexpansive mapping of $\left(M, \rho_{f}\right)$ into itself which maps $M_{0}^{f}$ isometrically onto itself.

ProOF. For each integer $i \geq 0$, let

$$
M_{i}^{f}= \begin{cases}\bigcap_{m \geq 0} f^{m}(M) & \text { if } i=0, \\ f^{-i}\left(M_{0}^{f}\right) \backslash f^{-(i-1)}\left(M_{0}^{f}\right) & \text { if } i=0 .\end{cases}
$$

By (4.2) and (13), $f\left(M_{0}^{f}\right)=M_{0}^{f}$ and $f\left(M_{i}^{f}\right) \subset M_{i-1}^{f}$ for each $i>0$ and the sets $M_{i}^{f}, i \geq 0$, are disjoint, open and closed subsets of $M$. Since $M$ is compact and $M_{0}^{f}=\bigcap_{m \geq 0} f^{m}(M)$ is open, there exists an integer $m \geq 0$ such that $M_{0}^{f}=$ $f^{m}(M)$. Let $n=\min \left\{m \geq 0: f^{m}(M)=M_{0}^{f}\right\}$, then the sets $M_{i}^{f}, i=0, \ldots, n$, cover $M$ and if $1 \leq i \leq n$ then $M_{i}^{f} \neq \emptyset$.

This proves the existence of the decomposition (10) satisfying (11) and (12). For the uniqueness, it is sufficient to observe that for each decomposition (10), conditions (11) and (12) imply (13).

The last statement of the theorem follows from (3.3), hence the proof is complete. 
We note the following:

(4.4) COROLLARY. Let $f$ be a local isometry of a compact metric space $(M, \rho)$ into itself. Then the following are equivalent:

(i) $f$ is injective,

(ii) $f$ is surjective,

(iii) $f$ is a homeomorphism of $M$ onto itself.

Further, if $f$ is a nonexpansive local isometry, those conditions are equivalent to (iv) $f$ is an isometry.

Proof. The proof follows from (4.3), since each of (i)-(iii) is equivalent to $M_{0}^{f}=M$, while if $f$ is nonexpansive, then by (7), $\rho_{f}=\rho$.

5. Some consequences. For local isometries on continua we obtain

(5.1) THEOREM. Let $f$ be a local isometry of a metric continuum $(M, \rho)$ into itself and let $\rho_{f}$ be the induced metric on $M$. Then $\rho_{f}$ is a metric on $M$ such that $\rho_{f}$ and $\rho$ are locally identical and $f$ is an isometry of $\left(M, \rho_{f}\right)$ onto itself.

Proof. The proof follows from (4.3) since, $M$ is connected implies $M_{0}^{f}=M$.

We have the following immediate corollaries of (5.1) and (4.4):

(5.2) COROLLARY. Each local isometry of a metric continuum into itself is a homeomorphism onto itself.

(5.3) COROLLARY. Each nonexpansive local isometry of a metric continuum into itself is an isometry onto itself.

The convexity in the next result is to be understood in the sense of Menger [8] (cf. also [1]). A metric space $(M, \rho)$ is convex if for each two distinct points $x, y \in M$ there exists a point $z \in M, z \neq x, y$, such that $\rho(x, y)=\rho(x, z)+\rho(z, y)$.

The following fact is well known and may be found in $[4, \S 3]$ :

(5.4) LEMMA. Each locally nonexpansive mapping of a convex metric continuum into itself is (globally) nonexpansive.

(5.5) THEOREM. Each local isometry of a convex metric continuum into itself is an isometry onto itself.

PROOF. The proof follows from (5.3) and (5.4).

\section{REFERENCES}

1. L. E. Blumenthal, Theory and applications of distance geometry, Clarendon Press, Oxford, 1953.

2. H. Busemann, The geometry of geodesics, Academic Press, New York, 1955.

3. - Geometries in which the planes minimize area, Ann. Mat. Pura Appl. 55 (1961), 171-189.

4. M. Edelstein, An extension of Banach's contraction principle, Bull. Amer. Math. Soc. 12 (1961), 7-10.

5. H. Freudenthal and W. Hurewicz, Dehnungen, Verkürzungen, Isometrien, Fund. Math. 26 (1936), 120-122.

6. W. A. Kirk, On conditions under which local isometries are motions, Colloq. Math. 22 (1971), 229-232.

7. A. Lindenbaum, Contributions d l'étude de l'espace métrique. I, Fund. Math. 8 (1926), 209-222.

8. K. Menger, Untersuchungen über allgemeine Metric, Math. Ann. 100 (1928), 75-163.

9. W. Nitka, Bemerkungen über nichtisometrische Abbildungen, Colloq. Math. 5 (1957), $28-31$.

10. J. Szenthe, Über metrische Raume, deren localisometrische Abbildungen Isometrien sind, Acta Math. Acad. Sci. Hungar. 13 (1962), 433-441.

Mathematical Institute, WroctaW UNIVERsity, 50-384 WrocŁaW, POLAND 